\title{
Stacked DIEP and Implant for Unilateral Breast Reconstruction
}

\author{
Elsie M. Rodebeck, MD ${ }^{1}$ Craig A. Blum, MD ${ }^{2}$ Frank J. DellaCroce, MD² \\ ${ }^{1}$ Department of Breast Microsurgery, Tulane University School of \\ Medicine, New Orleans, Louisiana \\ ${ }^{2}$ Center for Restorative Breast Surgery, St. Charles Surgical Hospital, \\ New Orleans, Louisiana \\ Address for correspondence Craig A. Blum, MD, Center for \\ Restorative Breast Surgery, St. Charles Surgical Hospital, \\ 1717 St. Charles Avenue, New Orleans, LA 70130 \\ (e-mail: drb@breastcenter.com).
}

J Reconstr Microsurg Open 2017;2:e124-e125.

The Deep Inferior Epigastric Perforator (DIEP) flap is the preferred method of autogenous breast reconstruction as it provides an ideal replacement for the absent breast tissue with minimal donor site morbidity and improved abdominal contour. When a single hemiabdomen is inadequate for unilateral reconstruction the volume and projection can be augmented with an implant, or the experienced microsurgeon may offer stacked DIEP flaps. ${ }^{1}$ An additional consideration would be the use of both hemiabdominal flaps in addition to an implant. This hybrid combination could maximize the autogenous contribution to the reconstruction allowing the use of a smaller implant. Additionally, total implant coverage with stacked DIEP flaps could help prevent rippling, improve projection, and lessen implant palpability. We describe two cases of hybrid reconstruction combining stacked DIEP flaps with immediate silicone implantation to improve reconstructive outcomes.

\section{Cases}

Ms. DD is a 60-year-old woman with a history of left breast cancer who underwent left mastectomy (875 g) without immediate reconstruction and right-sided reduction mammoplasty. She then received adjuvant radiotherapy to the left chest and subsequently underwent delayed hybrid stacked DIEP flap plus implant breast reconstruction. A 120cc moderate profile silicone implant was placed atop the pectoralis muscle and secured to the chest wall with acellular dermal matrix (ADM). The left hemiabdominal flap (490 g) was deepithelialized and placed atop the superior portion of the implant filling the upper pole of the breast. The right flap ( $505 \mathrm{~g}$ ) served as the superficial flap covering the lower portion of the implant and partly overlapping the deep flap for improved projection. An antegrade/retrograde perfusion pattern was created and both flaps were well-perfused providing thick and complete autogenous implant coverage.
Ms. AA is a 62-year-old woman with a history of left breast cancer who underwent left mastectomy, immediate implant reconstruction, and right breast augmentation at an outside facility. After multiple revisions she remained unsatisfied with her result. She presented with breast asymmetry and thinning of the breast skin with obvious rippling (-Fig. 1). A stacked DIEP flap plus implant hybrid was used to reconstruct her breast. A 500cc moderate profile implant was removed and a $158 \mathrm{cc}$ moderate-plus implant was placed atop the pectoralis muscle and secured to the chest wall with ADM. The left hemiabdominal flap (200 g) was deepithelialized and inset dermis side up to reconstruct the lower pole and cover the inferior aspect of the implant. The right hemiabdominal flap (205 g) was also deepithelialized and inset dermis side up to reconstruct the upper pole of the breast and cover the superior aspect of the implant. A branch chain perfusion pattern was successfully created and the flaps were sutured to one another as they lay side by side providing total implant coverage; eliminating the upper pole rippling (-Fig. 2).

\section{Discussion}

A single DIEP flap often provides sufficient tissue for unilateral reconstruction, but may be inadequate to reconstruct larger breasts in patients with leaner abdomens. ${ }^{1-4}$ In such cases, as the two presented above, several options may be considered including primary implant augmentation of a single DIEP flap. ${ }^{2,3}$ Figus et al. describes 14 such cases of subpectoral implants placed primarily at the time of DIEP reconstruction with a reported average of $20 \%$ or $1: 5$ implant to flap weight ratio. The authors suggest that the implant primarily contributes to breast volume, while shape and projection depends mostly on the abdominal tissue. ${ }^{2}$ The procedure is less technically demanding than stacked flaps, but there is an associated risk of implant-related received

June 10, 2017

accepted after revision

July 27,2017
DOI https://doi.org/

$10.1055 / \mathrm{s}-0037-1606355$. ISSN 2377-0813.
Copyright $\odot 2017$ by Thieme Medical Publishers, Inc., 333 Seventh Avenue, New York, NY 10001, USA. Tel: +1(212) 584-4662.
License terms

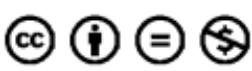




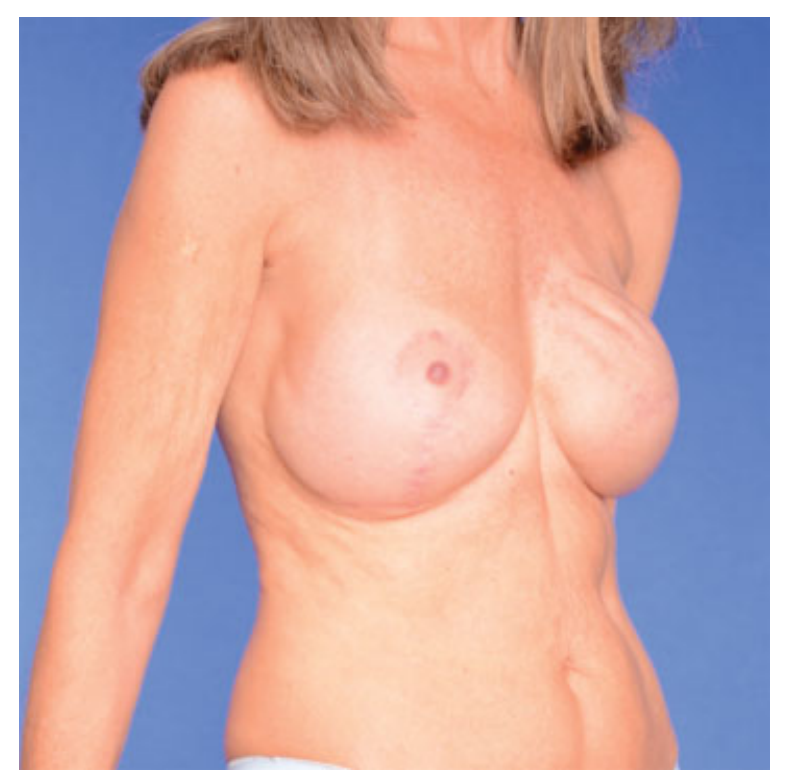

Fig. 1 Preoperatively, the upper pole of patient AA's reconstructed left breast demonstrates visible rippling secondary to the thin mastectomy flap skin and lack of soft tissue coverage.

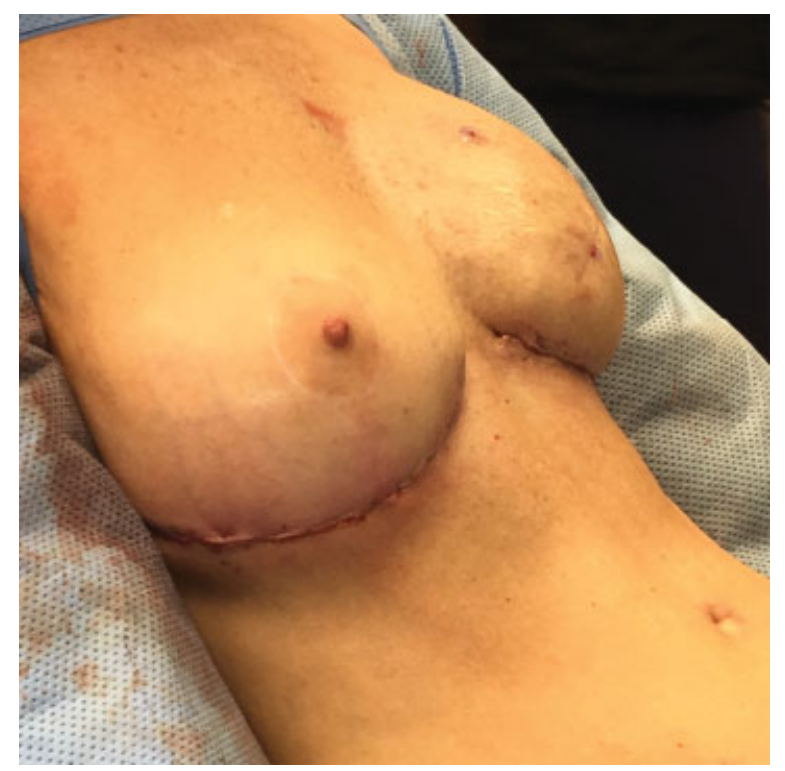

Fig. 2 Immediate postoperative result of stacked DIEP flap plus implant hybrid breast reconstruction. Note the improvement in the left upper pole eliminating the visible rippling.

complications such as infection or malpositioning which could lead to vascular compromise if the implant impinges on the flap pedicle.

Another option when a single DIEP flap fails to provide sufficient tissue for unilateral reconstruction, includes the sequential linkage and stacked inset of two individual DIEP flaps as described by DellaCroce et al. While a lengthier and more technically difficult operation, stacked DIEP reconstruction has many benefits. Perhaps it's greatest benefit is geometric versatility. Tailoring of inset patterns can achieve the best possible breast shape and symmetry for the patient. ${ }^{1,4}$ In addition it takes advantage of low donor site morbidity, often improves abdominal contour, and has been demonstrated to have excellent, reproducible aesthetic outcomes and patient satisfaction.

Recently, we have had success combining these two approaches using a stacked DIEP plus implant hybrid reconstruction. In each case we felt the patient would benefit from the additional volume and projection offered by a wellplaced silicone implant but we also desired thick and complete autogenous implant coverage to prevent visible rippling and lessen implant palpability. We chose to the place the implant in the pre-pectoral position just adjacent to the lateral border of the internal mammary access site to maximize projection. We carefully secured the position of the implant with ADM paying particular attention to suture the medial border of the ADM to the perichondrium of the 4th cartilaginous rib defect to prevent medial migration. We accepted the cost associated with the ADM because of the security of implant positioning that it afforded. The hybrid construct takes advantage of the stacked DIEP's inset pattern versatility including arrangements such as the side by side inset used in AA's case versus a partially overlapping inset as in DD's case. These inset patterns were chosen to ensure complete implant coverage and improve projection where necessary for each patient, all while maintaining flexibility in vascular construct arrangement.

\section{Conclusion}

Stacked DIEP flap and implant reconstruction is a reasonable option for cases when adequate volume and projection cannot be obtained with stacked DIEP reconstruction alone. This approach combines the implant's volume and projection augmentation with the stacked-DIEP's inset pattern versatility to ensure total implant coverage while simultaneously tailoring breast shape and projection for optimal, individualized aesthetic outcomes.

\section{Conflict of Interest}

None.

\section{References}

1 DellaCroce FJ, Sullivan SK, Trahan C. Stacked deep inferior epigastric perforator flap breast reconstruction: a review of 110 flaps in 55 cases over 3 years. Plast Reconstr Surg 2011;127(03): 1093-1099

2 Figus A, Canu V, Iwuagwu FC, Ramakrishnan V. DIEP flap with implant: a further option in optimising breast reconstruction. J Plast Reconstr Aesthet Surg 2009;62(09):1118-1126

3 Roehl KR, Baumann DP, Chevray PM, Chang DW. Evaluation of outcomes in breast reconstructions combining lower abdominal free flaps and permanent implants. Plast Reconstr Surg 2010;126 (02):349-357

4 Patel NG, Rozen WM, Chow WT, et al. Stacked and bipedicled abdominal free flaps for breast reconstruction: considerations for shaping. Gland Surg 2016;5(02):115-121 